PAPER

\title{
Modafinil for daytime somnolence in Parkinson's disease: double blind, placebo controlled parallel trial
}

\author{
W G Ondo, R Fayle, F Atassi, J Jankovic
}

J Neurol Neurosurg Psychiatry 2005;76:1636-1639. doi: 10.1136/jnnp.2005.065870

See end of article for authors' affiliations

.....................

Correspondence to: Dr W Ondo, 6550 Fannin, Ste 1801, Houston, TX 77030, USA; wondo@ bcm.tmc.edu

Received 17 February 2005 Revised version received 29 April 2005

Accepted 3 May 2005

\begin{abstract}
Background: Excessive daytime somnolence (EDS) commonly complicates Parkinson's disease (PD). The aetiology of EDS is probably multifactorial but is probably exacerbated by dopaminergic medications. Modafinil is a wake-promoting agent approved for use in narcolepsy, but it is often used to treat a variety of somnolent conditions.

Method: A double blind, placebo controlled parallel design trial was conducted to assess the efficacy of modafinil (200-400 mg/day) for the treatment of EDS in PD. The primary efficacy measure was the Epworth Sleepiness (ES) scale score. Secondary efficacy points included the Unified Parkinson's Disease Rating Scale (UPDRS), the Fatigue Severity Scale, the Hamilton Depression Scale, and the multiple sleep latency test (MSLT).

Results: Of a total of 40 subjects (29 men, mean (SD) age 64.8 (11.3) years), randomised to modafinil or placebo, 37 completed the study. Modafinil failed to significantly improve ES scores compared with placebo (2.7 $\vee 1.5$ points improvement, respectively, $p=0.28$ ). MSLT failed to improve with modafinil relative to placebo $(-0.16 v-0.70$, respectively, $p=0.14)$. UPDRS, global impressions, Fatigue Severity Scale, and Hamilton Depression Scale scores were unchanged. Adverse events were minimal.

Conclusion: Modafinil failed to significantly improve EDS in PD compared with placebo. The drug did not alter motor symptoms in PD and was well tolerated.
\end{abstract}

$\mathrm{E}$ xcessive daytime somnolence (EDS) occurs frequently in patients with Parkinson's disease (PD). In our evaluation of 303 consecutive PD patients seen at the Baylor College of Medicine, we found that $50.2 \%$ reported pathological sleepiness with Epworth Sleepiness (ES) scores of greater than $10 .^{1}$ The aetiology of EDS is probably multifactorial, but in our series EDS correlated with worse PD, a longer duration of $\mathrm{PD}$, male sex, and the use of dopamine agonists. Other reports and series have confirmed the frequent occurrence of EDS and other sleep problems in $\mathrm{PD}^{2-9}$ and reduced levels of hypocretin in cerebrospinal fluid. ${ }^{10}$ Although disturbed nocturnal sleep is common in PD, it does not usually correlate with EDS. ${ }^{811}$

Modafinil is a wake-promoting agent originally developed for the treatment of narcolepsy. ${ }^{12-14}$ It has also been shown to potentially improve wakefulness in a variety of neurological conditions including PD. ${ }^{15-21}$ This broad spectrum of clinical action, including diseases of primary somnolence (narcolepsy) and somnolence secondary to poor nocturnal sleep (sleep apnoea), suggests that its effects are not specific to the aetiology of the EDS. The mechanism of action is not clearly understood because the drug has little affinity for most major neurotransmitter receptors including dopamine. Modafinil mostly binds in the amygdala and anterior hypothalamus, ${ }^{22}$ including the tuberomammillary nucleus. ${ }^{23}$ On the basis of the well documented antisoporific effects of modafinil (Provigil; Cephalon, Frazer, PA), we designed a double blind, placebo controlled parallel trial to determine whether modafinil is effective in reversing daytime sleepiness in patients with PD.

\section{METHODS}

The Baylor College of Medicine institutional review board approved the protocol. We recruited patients meeting the diagnosis of PD from the Baylor College of Medicine Parkinson's Disease Center and Movement Disorders Clinic. All subjects satisfied the diagnostic criteria for $\mathrm{PD}^{24}$ were between 35 and 80 years of age, and reported daytime somnolence as measured by an ES score of greater than $10 .^{25}$ Patients with serious medical conditions, known narcolepsy, known sleep apnoea, and pregnancy were excluded. The subjects were not allowed to take prescription stimulant medications.

We collected demographic data, including a composite dopaminergic dose using the formula: dose $=$ levodopa $/ 100$ + controlled release levodopa/130 + pramipexole/ $1+$ pergolide/0.75 + ropinirole/3.5. If entacapone or tolcapone was used, we increased the levodopa dose by $10 \%$. Other PD medications were not included in the formula.

The primary efficacy point was a change in ES score, as we feel that this best captures the daytime sleep problems experienced by most patients with PD. Secondary endpoints included the Unified Parkinson's Disease Rating Scale (UPDRS), ${ }^{26}$ the Fatigue Severity Scale, ${ }^{27}$ the Hamilton Depression Scale, ${ }^{28}$ global impressions, and the Medical Outcome Survey Short Form 36 (SF-36) Quality of Life scale. ${ }^{29}$ We also systematically assessed adverse events. The subjects also underwent a standard multiple sleep latency test (MSLT) in the morning (naps at $9 \mathrm{am}, 11 \mathrm{am}, 1 \mathrm{pm}$, $3 \mathrm{pm})$ at baseline and after treatment. ${ }^{30}$ Sleep onset was scored at the first epoch of any identifiable stage of sleep after lights out. Subjects also completed a sleep survey, including their subjective report of the previous nights. Those with motor fluctuations also completed a one day "on/off" diary just before starting the study drug and one day before their final evaluation. The UPDRS part III motor examinations were done in the "on" state. If subjects were fluctuators, the UPDRS part II activities of daily living scores were calculated by averaging the "on" and "off" scores.

Abbreviations: EDS, excessive daytime somnolence; ES, Epworth Sleepiness (score); MSLT, multiple sleep latency test; PD, Parkinson's disease; UPDRS, Unified Parkinson's Disease Rating Scale 
Table 1 Demographic and entry data $(n=40)$

\begin{tabular}{|c|c|c|c|}
\hline & Modafinil & Placebo & Entire group \\
\hline & $(n=20)$ & $(n=20)$ & $(n=40)$ \\
\hline Age (years)* & $64.4(10.4)$ & $65.1(12.3)$ & $64.8(11.3)$ \\
\hline Sex (men/women) & $13 / 7$ & $13 / 7$ & $29 / 11$ \\
\hline $\begin{array}{l}\text { Duration of Parkinson's } \\
\text { disease }^{*}\end{array}$ & $6.5(5.5)$ & $7.0(4.6)$ & $6.8(5.0)$ \\
\hline $\begin{array}{l}\text { Dopaminergic dose } \\
(\mathrm{mg} / \text { day })^{*}\end{array}$ & $7.3(3.5)$ & $9.5(5.2)$ & $8.5(4.6)$ \\
\hline Fluctuating response & $5 / 20$ & $7 / 20$ & $12 / 40$ \\
\hline $\begin{array}{l}\text { UPDRS activities of daily } \\
\text { living*}\end{array}$ & $12.9(5.5)$ & $14.4(6.0)$ & $13.7(5.8)$ \\
\hline UPDRS motor ${ }^{*}$ & $24.1(9.8)$ & $29.2(9.5)$ & $26.7(9.8)$ \\
\hline Epworth score* & $15.8(3.0)$ & $15.9(3.5)$ & $15.8(3.2)$ \\
\hline
\end{tabular}

After signing informed consent and baseline assessments including the MSLT, the subjects were randomised by a computerised randomisation code to receive either modafinil or placebo in a 1:1 ratio. Both the drug and the placebo, which matched the drug in taste and appearance, were supplied by Cephalon Inc. and distributed to the coordinator by another coordinator who was shielded from the subjects and not otherwise involved in the study in any way. The subjects began taking modafinil, one pill of $100 \mathrm{mg}$, or matching placebo, upon waking and at lunch (200 mg/day). After one week, the dose was increased to two pills twice a day $(400 \mathrm{mg} /$ day). One week later, we administered the ES and queried the subjects by phone interview about adverse events. If a subject had experienced adverse events at the higher dose, they were allowed to decrease the medication to the previous dose. The rating physician was blinded to reports of adverse events and dosing. Subjects continued at either $200 \mathrm{mg} /$ day or $400 \mathrm{mg} /$ day until the second visit, four weeks after the initial visit. This consisted of an identical assessment to the first, including MSLT.

\section{Statistical analysis}

The primary efficacy analysis was an intention to treat design and included data from all enrolled subjects who had a baseline and at least one post-baseline efficacy measurement, including the safety check and ES done via phone. All efficacy analyses compared the efficacy variables to their baseline values. We analysed the change from baseline at all scheduled visits and last visit in the above mentioned efficacy variables using an analysis of covariance (ANCOVA) model with treatment and baseline values in the model. The proportion of Fatigue Severity Scale and global impression responders were analysed using a $\chi^{2}$ test or Fisher's exact test if warranted. The global impression response at the last visit was analysed using the Cochran-Mantel-Haenszel test. The normality assumption was examined for each continuous efficacy variable. If the assumption was not satisfied ( $p$ value from the Shapiro-Wilks test $\leqslant 0.10$ ), equivalent non-parametric techniques were applied on the variable. Specifically, a non-parametric ANCOVA was performed using the rank scores for the change from baseline scores and the baseline values in the model. All statistical comparisons were two tailed with a level of significance set at $\alpha=0.05$. For all the ANCOVA, we used type III sums of squares for the statistical inference.

We conducted a power analysis to determine the minimum sample size needed to detect a four point ES score difference between two groups. Based on the mean (SD) of qualifying subjects $(E S>10)$ from our survey population of 303 patients with $\mathrm{PD},{ }^{1}$ we required a total of 28 ( 14 per group) participants to achieve a power of 0.81 at $\alpha=0.05$ (two tailed).

\section{RESULTS}

There were no significant differences in any demographic or baseline variables between the subjects assigned to the study drug and placebo (table 1). Three subjects dropped out: two men, both of whom were taking placebo (one due to acute illness and subsequent death from acute myelogenous leukaemia and the other due to his spouse's serious illness that prevented his return), and one woman, who was on modafinil (who stopped due to instructions by her local physician to stop "study medication" because of back pain). All three dropped out prior to any post-drug evaluation. The remaining 37 patients completed all assessments.

There was no significant change in the primary endpoint, the ES score. Subjects on modafinil showed an improvement of 2.7 points compared with those on placebo who improved by 1.5 points $(p=0.28)$. MSLT results were not significantly different although the scores worsened less with modafinil $(-0.16$ (3.59) minutes) than with placebo $(-0.70$ (3.28) minutes), $\mathrm{p}=0.14$ (table 2). The UPDRS, Fatigue Severity Scale, Hamilton Depression Scale, SF-36, and global impression scores did not significantly change compared to

Table 2 Efficacy data $(n=37)^{*}$

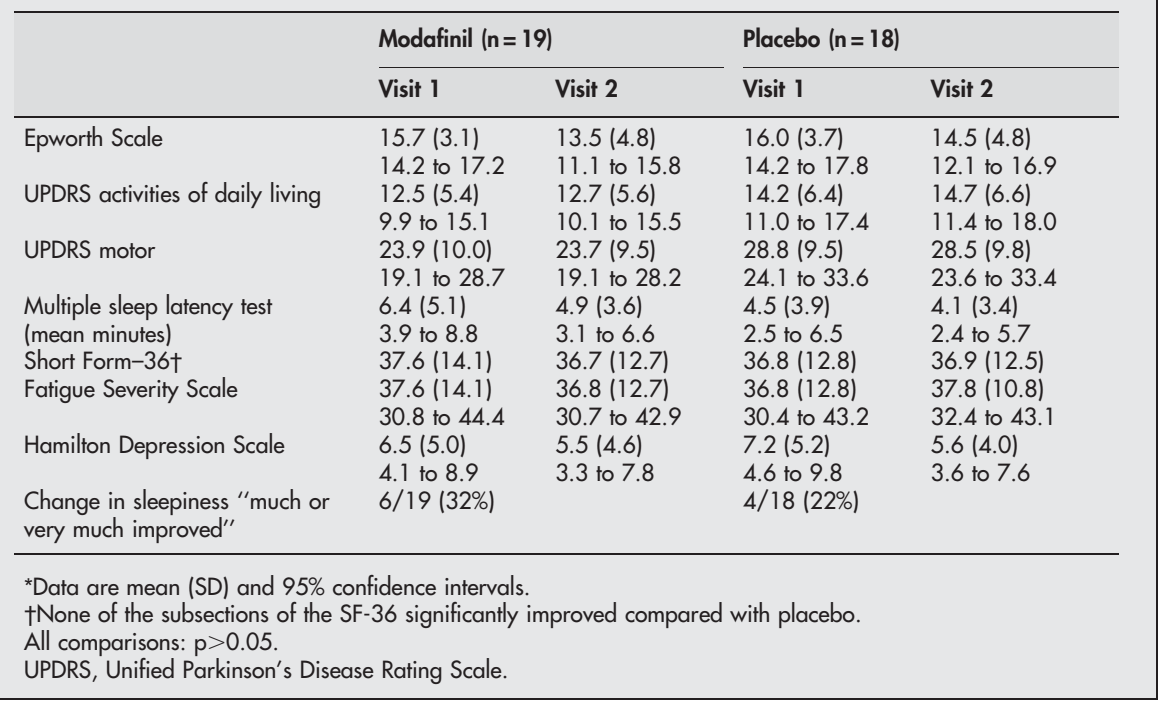


placebo. In fluctuating subjects, there was no change in on/ off time (table 2).

The medication was well tolerated in our patients. Only one patient taking modafinil elected to return to the lower dose, secondary to nausea and anxiety. Other adverse events thought to be at least possibly related to drug included dry mouth $(\mathrm{n}=1)$, dizziness $(\mathrm{n}=1)$, and back pain $(\mathrm{n}=1)$. Adverse events recorded in subjects taking placebo included hypotension requiring hospitalisation and reduction of antihypertensive medications $(\mathrm{n}=1)$, renal calcinosis $(\mathrm{n}=1)$, and blurred vision $(\mathrm{n}=1)$.

\section{DISCUSSION}

This double blind, placebo controlled study of patients with PD failed to show a significant reduction of daytime somnolence as measured by ES, the primary endpoint. Secondary outcome measures such as MSLT, Fatigue Severity Scale, Hamilton Depression Scale, and global impression scores also showed no difference between modafinil and placebo. The PD motor status was not affected and adverse events were minimal.

These results contrast with the results of two other controlled trials that have reported significantly improved ES in patients with EDS associated with PD. ${ }^{20}{ }^{21}$ Both trials were crossover, smaller, and of shorter duration, although Adler et al's study was re-analysed as a parallel design using the first arm due to a large carry-over effect. Neither reported a power analysis. Patient demographics (age, sex ratio, duration of PD) were similar to our study, although baseline sleepiness was less severe in Hogl et al's study. ${ }^{20}$ The dose of modafinil in both studies was actually lower than in ours. The actual improvement in ES on drug was 2.7 in our study compared with 3.3 and 3.4 in the others. The placebo response was also slightly greater in our study resulting in a smaller overall treatment effect (1.2 in ours $v 2.6$ and 4.7 in the others). The greater placebo response may have resulted from the twice daily dosing.

The PD subjects enrolled in our study demonstrated marked EDS as determined by MSLT and ES. Both measures were similar in severity to those seen in narcolepsy patients. ${ }^{25} 3132$ In contrast with narcolepsy, however, only four of our subjects demonstrated any early onset rapid eye movement period (EOREMP). In those four subjects, 9/32 nap opportunities $(28 \%)$ resulted in EOREM. These findings concur with some other reports, which do not show EOREMP ${ }^{33}{ }^{34}$ but contrast with others that show moderate rates of EOREM, still lower than that seen with narcolepsy. ${ }^{11}$

Several other potential methodological issues related to our study should be addressed. Firstly, we are a tertiary referral centre and our PD patient population may differ from that in a primary care setting. Secondly, polysomnographic testing on the night before MSLT would have been ideal to better interpret the MSLT data and evaluate for sleep apnoea or other specific nocturnal problems that could affect daytime somnolence. Nevertheless, in our PD population, the subjective reports of the two groups regarding their previous night's sleep were similar, and we have no reason to hypothesise that the two randomised groups would have had different polysomnogram results. The Maintenance of Wakefulness Test ${ }^{35}$ may have more sensitively captured a pharmacological intervention for wakefulness ${ }^{36}$; however, we felt that the MSLT best reflects the clinical scenario seen in PD. Furthermore, the only study that measured Maintenance of Wakefulness Test in PD patients also failed to show significant improvement with modafinil over placebo. ${ }^{20}$ Thirdly, employing a minimum cut-off of the primary variable (ES) in the exclusion criteria can result in artificial regression toward the mean. Three subjects on placebo reported a greater than five point improvement in ES. Our subjects only took $200 \mathrm{mg}$ modafinil or placebo prior to their MSLT, as they took their second dose after its completion. Lastly, despite our power analysis, this was still a relatively small study and may suffer from type II error. It was also a short term study.

\section{CONCLUSION}

Our results do no support the efficacy of modafinil (400 mg/ day in dived doses) for daytime somnolence in PD. The drug, however was very well tolerated and has an immediate effect, and individual patients did benefit from taking it. Since the aetiology of excessive sleepiness is multifactorial, modafinil may be considered on an individual basis. Furthermore, the equipoise generated by mixed study results justifies additional trials.

\section{ACKNOWLEDGEMENTS}

We would like to acknowledge the assistance of J Ernesto Jimenez, Med, and Kevin Dat Vuong, MA.

\section{Authors' affiliations}

W G Ondo, F Atassi, J Jankovic, Baylor College of Medicine, Houston, TX, USA

R Fayle, Park Plaza Hospital, Houston, TX, USA

This study was funded by an unrestricted educational grant from Cephalon Pharmaceuticals, the makers of Provigil

Competing interests: W Ondo spoke for Cephalon on several occasions approximately two years previously

\section{REFERENCES}

1 Ondo WG, Dat Vuong K, Khan H, et al. Daytime sleepiness and other sleep disorders in Parkinson's disease. Neurology 2001;57:1392-6.

2 Frucht SJ, Greene P, Fahn S. Sleep episodes in Parkinson's disease: a wake-up call. Mov Disord 2000;15:601-3.

3 Hobson DE, Lang AE, Martin WR, et al. Excessive daytime sleepiness and sudden-onset sleep in Parkinson disease: a survey by the Canadian Movement Disorders Group. JAMA 2002;287:455-63.

4 Horiguchi J, Inami Y, Nishimatsu O, et al. Sleep-wake complaints in Parkinson's disease. Rinsho Shinkeigaku 1990;30:214-16.

5 van Hilten JJ, Weggeman $M$, van der Velde EA, et al. Sleep, excessive daytime sleepiness and fatigue in Parkinson's disease. J Neural Trans Park Dis Dement Sect 1993;5:235-44.

6 Montastruc JL. Sleep attacks and antiparkinsonian drugs: a pilot prospective pharmacoepidemiologic study. J Neurol Neurosurg Psychiatry 2001:70:727-33.

7 Tan E, Lum S, Fook-Chong S, et al. Evaluation of somnolence in Parkinson's disease: Comparison with age- and sex-matched controls. Neurology 2001;58:465-8.

8 Arnulf I, Konofal E, Merino-Andreu M, et al. Parkinson's disease and sleepiness: an integral part of PD. Neurology 2002;58:1019-24.

9 Razmy A, Lang AE, Shapiro CM. Predictors of impaired daytime sleep and wakefulness in patients with Parkinson disease treated with older (ergot) vs newer (nonergot) dopamine agonists. Arch Neurol 2004;61:97-102.

10 Drouot X, Moutereau S, Nguyen JP, et al. Low levels of ventricular CSF orexin/hypocretin in advanced PD. Neurology 2003;61:540-3.

11 Rye D, Bliwise D, Dihenia B, et al. Daytime sleepiness in Parkinson's disease. J Sleep Res 2000:9:63-9.

12 Littner M, Johnson SF, McCall WV, et al. Practice parameters for the treatment of narcolepsy: an update for 2000. Sleep 2001;24:451-66.

13 Mitler MM, Harsh J, Hirshkowitz M, et al. Long-term efficacy and safety of modafinil (PROVIGIL((R))) for the treatment of excessive daytime sleepiness associated with narcolepsy. Sleep Med 2000;1:231-43.

14 Randomized trial of modafinil as a treatment for the excessive daytime somnolence of narcolepsy: US Modafinil in Narcolepsy Multicenter Study Group. Neurology 2000;54:1166-75.

15 Happe S. Successful treatment of excessive daytime sleepiness in Parkinson's disease with modafinil. J Clin Psychol 2001;57:1559-69.

16 Hauser RA, Wahba MN, Zesiewicz TA, et al. Modafinil treatment of pramipexole-associated somnolence. Mov Disord 2000;15:1269-1271.

17 Rabinstein A, Shulman LM, Weiner WJ. Modafinil for the treatment of excessive daytime sleepiness in Parkinson's disease: a case report $2001 ; 7: 287-8$

18 Montastruc JL, Rascol O. Modafinil and pramipexole-associated somnolence. Mov Disord $2001 ; 16: 783-4$

19 Nieves AV, Lang AE. Treatment of excessive daytime sleepiness in patients with Parkinson's disease with modafinil. Clin Neuropharmacol 2002;25: $111-14$. 
20 Hogl B, Saletu M, Brandauer E, et al. Modafinil for the treatment of daytime sleepiness in Parkinson's disease: a double-blind, randomized, crossover, placebo-controlled polygraphic trial. Sleep 2002;25:905-9.

21 Adler CH, Caviness JN, Hentz JG, et al. Randomized trial of modafinil for treating subjective daytime sleepiness in patients with Parkinson's disease. Mov Disord 2003; 18:287-93.

22 Pal S, Bhattacharya K, Agapito C, et al. A study of excessive daytime sleepiness and its clinical significance in three groups of Parkinson's disease patients taking pramipexole, cabergoline and levodopa mono and combination therapy. J Neural Trans 2001;108:71-7.

23 Scammell TE, Estabrooke IV, McCarthy MT, et al. Hypothalamic arousal regions are activated during modafinil-induced wakefulness. J Neurosci 2000;20:8620-8

24 Gelb DJ, Oliver E, Gilman S. Diagnostic criteria for Parkinson disease. Arch Neurol 1999:56:33-9.

25 Johns MW. A new method for measuring daytime sleepiness: the Epworth sleepiness scale. Sleep 1991;14:540-5.

26 Martinez-Martin P, Gil-Nagel A, Gracia LM, et al. Unified Parkinson's Disease Rating Scale characteristics and structure. The Cooperative Multicentric Group. Mov Dis 1994;9:76-83.

27 Krupp LB. The fatigue severity scale. Application to patients with multiple sclerosis and systemic lupus erythematosus. Arch Neurol 1988;45:435-7.
28 Hamilton M. Development of a rating scale for primary depressive illness. Br J Soc Clin Psychol 1967;6:278-96.

29 Lyons RA. Evidence for the validity of the Short-form 36 Questionnaire (SF-36) in an elderly population. Age Ageing 1994;23:182-4.

30 Thorpy MJ. The clinical use of the Multiple Sleep Latency Test. The Standards of Practice Committee of the American Sleep Disorders Association. Sleep. 1992;15: 268-76, Erratum in Sleep, 1992;15:381

31 Guilleminault C. Controversies in the diagnosis of narcolepsy. Sleep 1994;17:619-23.

32 Association ASD. International classification of sleep disorders, revised: diagnostic and coding manual. Rochester, MN: American Sleep Disorder Association, 1997.

33 Tracik F. Sudden daytime sleep onset in Parkinson's disease: polysomnographic recordings. Mov Disord $2001 ; 16: 500-6$.

34 Hauser RA, Gauger L, Anderson WM, et al. Pramipexole-induced somnolence and episodes of daytime sleep. Mov Disord 2000;15:658-63.

35 Mitler MM. Maintenance of wakefulness test: a polysomnographic technique for evaluation treatment efficacy in patients with excessive somnolence. Electroencephalogr Clin Neurophysiol 1982;53:658-61.

36 van den Hoed J, Korner AF, Guilleminault C, et al. Disorders of excessive daytime somnolence: polygraphic and clinical data for 100 patients. Sleep $1981 ; 4: 23-37$.

\section{1 th European Forum on Quality Improvement in Health Care}

26-28 April 2006, Prague, Czech Republic

For further information please go to: www.quality.bmipg.com

Book early to benefit from a discounted delegate rate 